\title{
TAGUNG
}

\section{Die Gemeinschaftsgerichtsbarkeit in der erweiterten Union - Optimierung und Qualitätssicherung in einer transnationalen Rechtsgemeinschaft}

\author{
Carl Friedrich Nordmeier*
}

Die Erweiterung der Europäischen Union von 15 auf nunmehr 27 Mitgliedstaaten stellt die Gemeinschaftsgerichtsbarkeit vor neue Herausforderungen. Legitimation und Akzeptanz einer Rechtsgemeinschaft werden auch von der Durchsetzbarkeit der von ihr eingeräumten Rechte geprägt. Die Qualität der Rechtsverfolgung vor den europäischen Gemeinschaftsgerichten zu sichern und zu optimieren ist - vor allem vor dem Hintergrund der Erweiterung eine anspruchsvolle Aufgabe. Dem Begriff der , Rechtsstaatlichkeit" kommt in diesem Zusammenhang besondere Bedeutung zu. Ferner gilt es zu berücksichtigen, dass sich der Europäische Rat im Juni 2007 auf ein Mandat für eine Reform des Primärrechts geeinigt hat, sodass der ursprüngliche, Verfassungsvertrag zu neuem Leben erwacht.

Die Heidelberger Tagung verfolgte daher, wie Peter-Christian Müller-Graff in seiner BegrüBung und Einführung deutlich machte, zwei Ziele. Einerseits sollte eine Bestandaufnahme des Status quo der Gemeinschaftsgerichtsbarkeit erfolgen. Andererseits war eine Neuakzentuierung anzudenken, die vor dem Hintergrund der gewachsenen Zahl der Mitgliedstaaten erforderlich sein könnte.

Das Rechtsstaatlichkeitsprinzip auf der Ebene des Gemeinschaftsrechts

Als erster Referent analysierte Claus Dieter Classen die Bedeutung des Rechtsstaatlich-

\section{Gemeinschaftsgerichtsbarkeit und Rechtsstaatlichkeit in der erweiterten Union}

Wissenschaftliche Tagung des Arbeitskreises Europäische Integration e.V. in Zusammenarbeit mit dem Institut für deutsches und europäisches Gesellschafts- und Wirtschaftsrecht der Universität Heidelberg mit Unterstützung der Europäischen Kommission

Heidelberg, 13./14. Juli 2007

Wissenschaftliche Leitung

Prof. Dr. Dr. h.c. Peter-Christian MÜLLERGRAFF, Universität Heidelberg

Prof. Dr. Dieter H. SCHEUING, Universität Würzburg

Begrïßung und Einführung

Prof. Dr. Dr. h.c. Peter-Christian MÜLLERGRAFF, Universität Heidelberg

Rechtsstaatlichkeit als Primärrechtsgebot in der Europäischen Union: Vertragsrechtliche Grundlagen und Rechtsprechung der Gemeinschaftsgerichte

Prof. Dr. Claus Dieter CLASSEN, Universität Greifswald

Gemeinschaftsrichter und Generalanwälte als Akteure des gemeinschaftsrechtlichen Rechtsschutzes im Lichte des gemeinschaftsrechtlichen Rechtsstaatsprinzips

Prof. Dr. Jörg GUNDEL, Universität Bayreuth

Rechtsstaatliche Vorzüge und Mängel der Verfahren vor den Gemeinschaftsgerichten

Prof. Dr. Bernhard W. WEGENER, Universität

Erlangen

* Carl Friedrich Nordmeier, Assistent am Institut für Deutsches und Europäisches Gesellschafts- und Wirtschaftsrecht (Lehrstuhl Prof. Dr. Dr. h.c. Peter-Christian Müller-Graff), Universität Heidelberg, und am Institut für Ausländisches und Internationales Privat- und Wirtschaftsrecht (em. Prof. Dr. Dr. h.c. mult. Erik Jayme, LL. M.), Universität Heidelberg. 
keitsbegriffs in der Europäischen Union. Er wies auf die unterschiedliche Konkretisierung dieses Begriffs in den Mitgliedstaaten hin, um sodann Regelungen des Vertrags zur Gründung der Europäischen Gemeinschaft (EGV) heranzuziehen, in denen das Rechtsstaatsprinzip zum Ausdruck komme. Die Betrachtung der Rechtsprechung veranlasste Classen zu der Beobachtung, dass das Bundesverfassungsgericht (BVerfG) den Begriff ,Rechtsstaatlichkeit" wesentlich öfter nutze als der Gerichtshof der Europäischen Gemeinschaften $(\mathrm{EuGH})$. Allerdings fände sich eine Vielzahl von Einzelfällen, in denen die Generalanwälte zur Begründung der Schlussanträge das Rechtsstaatsprinzip verwendeten. Der EuGH rekurriere vor allem dann auf das Prinzip, wenn er eine eigene Kontrollkompetenz eröffnen wolle, die sich nicht direkt aus dem Vertragstext ableiten ließe. Die Zurückhaltung in der Heranziehung des Rechtsstaatlichkeitsprinzips indiziere aber nicht, so Classen, einen Mangel an Rechtsstaatlichkeit auf Gemeinschaftsebene. Vielmehr ließe sich die große Mehrzahl der Ergebnisse auch durch Anwendung konkreter Rechtsinstitute erzielen, sodass ein Rückgriff auf das Rechtsstaatsprinzip vielfach nicht erforderlich sei.

In der folgenden Diskussion ging es um das Wesen des Rechtsstaatsprinzips als allgemeiner Rechtsgrundsatz der Gemeinschaft und sein Verhältnis zum Primär- und Sekundärrecht. Insbesondere stellte sich die Frage, ob das Rechtsstaatsprinzip selbst Teil des Primärrechts sei oder eher in einem Komplementärverhältnis zu diesem stehe.

Der Beitrag von Jörg Gundel widmete sich in einem ersten Teil den Gemeinschaftsrichtern. Für das Verfahren der Richter-Auswahl sei in Deutschland eine neue gesetzliche Bestimmung vorgesehen, deren Inkrafttreten aber an den Vertrag über eine Verfassung für Europa (VVE) gekoppelt wurde. Gesichert werde die richterliche Unabhängigkeit auf Gemeinschaftsebene durch die zeitliche Begrenzung der Amtszeit, Artikel 223 EGV, das Kollegialprinzip sowie das Beratungsgeheimnis. Zu-
Einstweiliger Rechtsschutz vor den Gemeinschaftsgerichten und vor den mitgliedstaatlichen Gerichten im Lichte des gemeinschaftsrechtlichen Rechtsstaatsprinzips

Prof. Dr. Alina-Maria LENGAUER, Universität Wien

Die kooperative Sicherung der Rechtsstaatlichkeit durch die mitgliedstaatlichen Gerichte und Gemeinschaftsgerichte aus mitgliedstaatlicher Sicht

Prof. Dr. Andreas HARATSCH, Universität Hagen

Die kooperative Sicherung der Rechtsstaatlichkeit durch die mitgliedstaatlichen Gerichte und Gemeinschaftsgerichte aus Sicht des EuGH Dr. Dieter KRAUS, Kabinettschef des Präsidenten des Gerichtshofs der Europäischen Gemeinschaften, Luxemburg

Binnendifferenzierung der Gemeinschaftsgerichtsbarkeit: Stärkung oder Schwächung der Rechtsstaatlichkeit der Europäischen Union? Prof. Dr. Adelheid PUTTLER, Universität Bochum

Befolgung und Durchsetzung der Urteile der Gemeinschaftsgerichte

Prof. Dr. Waltraud HAKENBERG, Kanzlerin des Gerichts für den öffentlichen Dienst, Luxemburg

Der Beitrag des Europäischen Gerichtshofs für Menschenrechte zur Sicherung der Rechtsstaatlichkeit der Europäischen Union

Dr. Johan CALLEWAERT, Vizekanzler der Großen Kammer des Europäischen Gerichtshofs für Menschenrechte, Straßburg

dem legte Gundel dar, dass die Anforderungen an ein „faires Verfahren“ in den Mitgliedstaaten divergieren. So sei die Zuweisung einer Rechtssache an einen Spruchkörper vor den europäischen Gerichten nicht so transparent wie in Deutschland. Auch überbesetzte Kammern seien problematisch.

Im zweiten Teil unterstrich Gundel die besondere Bedeutung der Schlussanträge der Generalanwälte für die Kohärenz der Entscheidungen. Die Schlussanträge hätten keinen Charakter von "dissenting opinions“. Dennoch stellten sie die persönliche Sicht des Generalanwalts dar, sodass umfangreiche Ver- 
weise im Urteil auf die Schlussanträge bedenklich seien. Auch würden die Parteien zu den Ansichten des Generalanwalts im Verfahren nicht gehört. Hier zeichnet sich ein Konflikt mit Artikel 6 der Europäischen Konvention zum Schutz der Menschenrechte und Grundfreiheiten (EMRK) ab, der jedoch nach Meinung des EuGH durch eine mögliche Wiedereröffnung des Verfahrens gelöst wird.

Die Diskussion zielte zunächst auf die unterschiedlichen Voraussetzungen zur Berufung als Richter am EuGH und am Gericht Erster Instanz (EuG) ab. Es folgten Hinweise auf die Vorschlagspolitik sowie den Konflikt zwischen dem Verlangen nach Transparenz einerseits und dem Schutz des richterlichen Beratungsgeheimnisses andererseits.

Effektiver und vorläufiger Rechtsschutz im Gemeinschaftsrecht

Bernhard W. Wegener nahm die Effektivität des Rechtsschutzes vor den Gemeinschaftsgerichten in den Blick. Er sprach sich für einen Vorrang des nationalen, das heißt dezentralen Rechtsschutzes aus, ohne jedoch den hierarchischen Aufbau mit Gemeinschaftsgerichten an der Spitze grundsätzlich in Frage stellen zu wollen. Wegener exemplifizierte dies an drei Punkten. Zunächst erfolgte eine Analyse der Schranken des Individualrechtsschutzes nach Artikel 230 Absatz 4 EGV. Problematisch sei die von der Norm geforderte ,individuelle Betroffenheit", welche den Rechtsschutz einenge. Die Rechtssuchenden kämen bei mangelnder individueller Betroffenheit zu den europäischen nur über die nationalen Gerichte. Zweiter Punkt war die auf Gemeinschaftsebene fehlende Nichtvorlagebeschwerde. Die Vorlage an die europäischen Gerichte könnte vor diesen nicht erzwungen werden, obgleich das Vorlageverfahren den dezentralen und zentralen Rechtsschutz in entscheidender Weise verknüpfe. $\mathrm{Zu}$ bedenken stehe jedoch, dass eine Nichtvorlagebeschwerde die Rechtskraft nationaler richterlicher Entscheidungen relativiere. Drittens kritisierte Wegener den numerus clausus der Klagearten vor den Gemeinschaftsgerichten. Dieser beeinträchtige die Effektivität des Rechtsschutzes. So müsste etwa eine Nichtigkeitsfeststellung zwar nach Artikel 233 EGV von den Organen beachtet werden. Im Übrigen bleibe aber Raum für eine neue Entscheidung.

Zum einstweiligen Rechtsschutz referierte Alina-Maria Lengauer. Ausgehend vom Rechtsstaatsprinzip erörterte sie dessen $\mathrm{Zu}$ sammenhang mit dem einstweiligen Rechtsschutz, um Vorgaben aufzuzeigen, die jenes für diesen aufstellt. Aus Artikel 220 EGV ergäben sich gewisse rechtsstaatliche Anforderungen. Insbesondere verlange die Norm, so Lengauer, Effektivität des Rechtsschutzes (effet utile). Vor diesem Hintergrund käme in Artikel 242f. EGV ein allgemeiner Rechtsgrundsatz der mitgliedstaatlichen Rechtsordnungen zum Ausdruck, nämlich dass durch die Verfahrensdauer die Rechtspositionen der Parteien nicht beeinträchtigt werden dürften. Es gelte zudem, die Wirksamkeit der Entscheidungen der Gemeinschaftsgerichte zu sichern. Auch diene der einstweilige Rechtsschutz der einheitlichen Anwendung des Gemeinschaftsrechts. Lengauer analysierte dann die Möglichkeit, einstweiligen Rechtsschutz gegen Gemeinschaftsakte vor nationalen Gerichten zu erlangen. Sie betonte dessen Akzessorietät zum Hauptsacheverfahren und gab zu bedenken, dass in die nationalen Rechtssysteme eingegriffen werde, wenn diese einen entsprechenden einstweiligen Rechtsschutz nicht kennen.

Kooperatives Zusammenwirken der mitgliedstaatlichen und der Gemeinschaftsgerichte

Das Zusammenspiel der Gemeinschaftsgerichte mit den nationalen Gerichten behandelte Andreas Haratsch. Er ging davon aus, dass einerseits die Union von den Mitgliedstaaten eine rechtsstaatliche Organisation fordere, andererseits aber auch die Mitgliedstaaten von der Union eine rechtsstaatliche Struktur verlangten. Für die deutschen Gerichte handele es sich bei unmittelbar anwendbarem Gemeinschaftsrecht um „Recht“ 
im Sinne von Artikel 20 Abs. 3 Grundgesetz. Normenkollisionen müssten vermieden werden, wobei der Vorrang des Gemeinschaftsrechts neben den Vorrang des Gesetzes trete. Der Nichtanwendung gemeinschaftsrechtswidrigen nationalen Rechts stellte Haratsch die Nichtanwendung grundgesetzwidrigen Gemeinschaftsrechts gegenüber. Im Hinblick auf ein jüngeres Urteil des Bundesarbeitsgerichts ${ }^{1}$ thematisierte er das Problem ausbrechender Rechtsakte.

Zudem sei effektiver Individualrechtsschutzes zu gewährleisten. Nationales Prozessrecht müsse so ausgelegt werden, dass effektiver Rechtsschutz gegen mitgliedstaatliche Umsetzungs- und Vollzugsakte erlangt werden könne. Hinzu trete das Vorabentscheidungsverfahren auf europäischer Ebene. Haratsch nannte außerdem die Möglichkeit der Verfassungsbeschwerde, gestützt auf Artikel 101 Abs. 1 S. 2 Grundgesetz, wenn nationale Gerichte vorlagepflichtige Fragen nicht vorlegten. Der Begriff des "gesetzlichen Richters“" werde hier gemeinschaftsrechtlich aufgeladen und durch den Gemeinschaftsrichter ergänzt. Die Kooperation zwischen den Gerichten der Mitgliedsstaaten und den Gemeinschaftsgerichten wurde anhand der ,Solange'-Rechtsprechung des BVerfG nochmals verdeutlicht.

Dieter Kraus sprach zur Sicherung der Rechtsstaatlichkeit aus der Perspektive des EuGH. Auch er betonte das Kooperationsmodell zwischen dem EuGH und den Gerichten der Mitgliedstaaten. Es bestehe keine Hierarchie, weil ein Rechtsmittel oder Rechtszug zum EuGH nicht existiere. Terminologisch grenzte Kraus den Begriff der Rechtsstaatlichkeit von dem der Rechtsgemeinschaft ab. Der Gerichtshof bevorzuge es, auf die Rechtsgemeinschaft zu rekurrieren, weil der Begriff der Rechtsstaatlichkeit im nationalen Bereich stark ausdifferenziert werde. Auch entwickle der EuGH eher anlassbezogen allgemeine
Grundsätze des Gemeinschaftsrechts, um rechtsstaatliche Grundsätze zu wahren. Ausgangspunkt sei meist die Wendung der „Wahrung des Rechts bei der Auslegung und Anwendung" der Verträge (Artikel 220 EGV).

Kraus behandelte die rechtsschutzfreundliche Handhabe des nationalen Verfahrensrechts, die etwa zur Schaffung zusätzlicher Rechtsbehelfe im nationalen Prozessrecht verpflichten könnte. ${ }^{2}$ Außerdem thematisierte er die rechtsschutzeröffnende Umqualifizierung von rechtsschutzlosen Akten der 3. Säule der Europäischen Union, welche Einzelpersonen in ihren Rechten beeinträchtigen. ${ }^{3}$ Daneben traten die Frage nach der Rücknahme bestandskräftiger, aber gemeinschaftsrechtswidriger nationaler Verwaltungsakte sowie nach dem Konflikt zwischen gemeinschaftsrechtlicher Beihilfenaufsicht und nationaler Rechtskraft.

Abschließend war Kraus der Meinung, dass durch die anstehende Reform des Primärrechts die Kooperation zwischen nationalen und europäischen Gerichten eine Stärkung erfahren werde. Insbesondere sei mit einer Schließung der Rechtsschutzlücken in der 2. und 3. Säule zu rechnen.

Die Problematik des gesetzlichen Richters auf Gemeinschaftsebene und der Gerichtsorganisation behandelte Adelheid Puttler. In Deutschland stelle das BVerfG strenge Anforderungen an die Sicherung des gesetzlichen Richters. Auf europäischer Ebene sei die Lage weniger transparent, was die Frage nach einem subjektiven Recht auf den gesetzlichen Gemeinschaftsrichter aufwerfe. Puttler unterschied zwischen dem EuGH und dem EuG. Es zeigten sich Differenzen in den Verfahrensordnungen und der Gerichtspraxis bezüglich der Besetzung der Kammern und der $\mathrm{Zu}$ weisungen der Verfahren an eine bestimmte Kammer. $\mathrm{Zu}$ einem Recht auf einen vorbestimmten Richter schwiegen sich die Verträge sowie Artikel 6 EMRK und Artikel 47 Abs. 2

\footnotetext{
BAG vom 26. April 2006 (Az. 7 AZR 500/04).

EuGH vom 13.03.2007, Rs. C-432/05 (Unibet).

EuGH vom 27.02.2007, Rs. C-354/04 P (Gestoras Pro Amnistía).
} 
der Europäischen Grundrechtecharta aus. Am ehesten könne ein solches als allgemeiner Rechtsgrundsatz aus den Rechten der Mitgliedstaaten hergeleitet werden. Rechtsvergleichend werde aber deutlich, dass auch in den Mitgliedstaaten erhebliche Divergenzen bestünden. Dennoch befürwortete Puttler ein subjektives Recht auf den gesetzlichen, das heißt vorbestimmten Richter. Je weiter sich die Gemeinschaftsgerichtsbarkeit ausdifferenziere, desto mehr Transparenz sei von Nöten.

Unterschiedliche Verfahrensarten verlangen unterschiedliche Vollzugsmechanismen

Im folgenden Referat differenzierte Waltraud Hakenberg zwischen verschiedenen Verfahrensarten, um die Durchsetzung der Urteile der Gemeinschaftsgerichte zu analysieren. In Vertragsverletzungsverfahren ergäbe sich die Pflicht zur Befolgung der Urteile aus Artikel 10 und 228 Abs. 1 EGV. Bei Nichtumsetzung könnten ein Zwangsgeld oder ein Pauschalbetrag verhängt werden, wobei der EuGH auch die Kumulation beider zuließe. ${ }^{4}$

Für die Nichtigkeits- und Untätigkeitsverfahren stellte Hakenberg fest, dass Urteile in Normkontrollverfahren in der Regel ohne Weiteres befolgt würden. In Verfahren unter Beteiligung Privater wache die Kommission über die Einhaltung der Urteile. Für Beamtensachen fokussiere das Gericht für den öffentlichen Dienst die Kontrolle der Exekutive, kassiere aber nur die Verwaltungsentscheidungen, das heißt ersetze sie nicht durch eigene. Da Beamte in einem besonderen Gewaltverhältnis stünden, sei ein Mechanismus zur Durchsetzung von Urteilen nicht nötig.

Das Vorabentscheidungsverfahren erlaube dem Gerichtshof nur, Auslegung beziehungsweise Normenkontrolle des Gemeinschaftsrechts für die nationalen Gerichte durchzuführen. Hakenberg thematisierte die Pflicht der Vorlagege- richte, die Rechtsmeinung des EuGH zu befolgen und leitete hieraus eine gewisse Präjudizwirkung der EuGH-Rechtsprechung nach Artikel 10 EGV her. Ein neuer Durchsetzungsmechanismus liege in der Haftung der Mitgliedstaaten für judikatives Unrecht. ${ }^{5} \mathrm{Au}-$ Berdem kamen zum einen die ,Rückwirkung " von Auslegungsurteilen und zum anderen die ,Wirkungsaussetzung ' zur Sprache. Hakenberg unterstrich abschließend die Bedeutung der Autorität der Gemeinschaftsgerichte.

Gemeinsame Sicherung der Rechtsstaatlichkeit durch EuGH und EGMR

Schließlich erörterte Johan Callewaert die Rolle des Europäischen Gerichtshofs für Menschenrechte (EGMR) für die gemeinschaftsrechtliche Rechtsstaatlichkeit. Diese werde durch die Rechtsprechung des EGMR beeinflusst, verstärkt, ergänzt, korrigiert und überprüft. Grundlage hierfür bilde ein besonderes Kooperationsverhältnis zwischen dem EuGH und dem EGMR. So sei für die Gemeinschaftsgerichte die EMRK in der Auslegung verbindlich, die sie durch den EGMR gefunden habe. ${ }^{6}$ Callewaert unterstrich, dass die EMRK von einer Rechtserkenntnisquelle zu einer Rechtsquelle des Gemeinschaftsrechts angewachsen sei. Zudem könne sie dazu dienen, Lücken im Rechtsschutzsystem der Gemeinschaft zu schließen. So sei eine Verletzung von Artikel 6 EMRK denkbar, wenn ein nationales Gericht seiner Vorlagepflicht aus Artikel 234 EGV nicht nachkomme. Letztlich habe die Konvention auch eine Korrekturwirkung. Im Zweifel müsse konventionswidriges Gemeinschaftsrecht hinter konventionskonformem mitgliedstaatlichen Recht zurücktreten. ${ }^{7}$ Dennoch sei das Verhältnis der EMRK zum Gemeinschaftsrecht keine „Einbahnstraße“. Vielmehr ließen sich auch Auswirkungen der Rechtsprechung des EuGH auf die Entscheidungen des EGMR nachweisen. Nach Callewaert bewegen sich

4 EuGH vom 12.07.2005, Rs. C-304/02 (Fischerei in der Bretagne).

5 EuGH vom 30.09.2003, Rs. C-224/01 (Köbler).

6 EuGH vom 16.06.2005, Rs. C-105/03 (Pupino).

7 EuGH vom 12.09.2006, Rs. C-145/04 (Gibraltar). 
beide Gerichte aufeinander zu und arbeiten an der Entstehung eines einzigen, gemeinsamen Grundrechtsraums.

In seinem Schlusswort stellte Müller-Graff Schnittmengen in zwei Bereichen fest, nämlich zum einen beim Kooperationsmechanismus und zum anderen bei der Garantie des gesetzlichen Richters. Letztlich genüge die Gemeinschaftsgerichtsbarkeit den Grundsätzen einer transnationalen Rechtsgemeinschaft.
Die Tagung machte deutlich, dass die Vorstellungen von Rechtsstaatlichkeit auf Gemeinschaftsebene in verschiedenen Punkten durchaus divergieren - wie auch die Methoden und Mittel, durch welche sie gesichert werden soll. Im Kern jedoch bleibt auch nach der Erweiterung der Union die legitimatorische Kraft der ,Rechtsstaatlichkeit“" für die Gemeinschaftsgerichtsbarkeit von zentraler Bedeutung.

\section{Aktuelle Neuerscheinung}

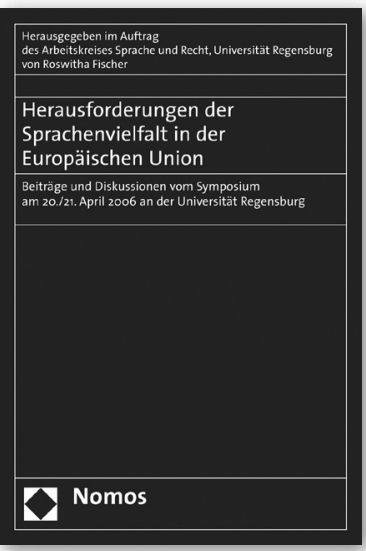

Herausforderungen der Sprachenvielfalt in der Europäischen Union

Beiträge und Diskussionen vom Symposium am 20./21. April 2006 an der Universität Regensburg Herausgegeben von Prof. Dr. Roswitha Fischer, Universität Regensburg 2007, 201 S., brosch., 45,-€, ISBN 978-3-8329-2698-4

Das Werk beschäftigt sich mit Erfahrungen, Problemen und Perspektiven der Sprachenvielfalt in der Europäischen Union. Er behandelt u. a. das Abfassen von Gesetzestexten, den Umgang mit Mehrsprachigkeit in den verschiedenen Einrichtungen der EU, Aspekte der europäischen Fremdsprachenpolitik sowie die Rolle einer einheitlichen Sprache. 